\title{
Student understanding of density: a cross-age investigation
}

\author{
R. E. Yeend, Science Education Program; M. E. Loverude, Department of Physics; \\ B. L. Gonzalez, Department of Chemistry and Biochemistry \\ California State University Fullerton, P.O. Box 6866, Fullerton, CA 92834
}

Abstract: Results of a density assessment administered to 787 middle school, high school, and college students indicate that students harbor numerous alternate conceptions, particularly a tendency to associate mass, volume, and density with size.

\section{INTRODUCTION}

Density is a basic concept that is a building block for further study in the sciences. It appears in science education standards as early as grades 5-8. ${ }^{1}$ However, most research on student understanding of density address the concept in the context of other topics, such as buoyancy. ${ }^{2}$ Lack of understanding of density has been shown to impact student learning of more advanced topics, from buoyancy to Gauss' law. ${ }^{3}$ This study seeks to probe student understanding of the most basic elements of density, independent of more sophisticated concepts.

The density of an object is defined as the mass per unit volume of the object. Deep understanding of density rests on underlying ideas including the concepts of mass and volume and the variation of density with each. Students are expected to know that density is a characteristic property of a substance, independent of sample size. Inhelder and Piaget attributed difficulties with density in part to difficulties with conservation of volume and proportional reasoning, and used density questions to test for formal reasoning ability. ${ }^{4}$

\section{METHODOLOGY}

The two purposes of the study are:

1. To examine student understanding of six fundamental aspects of density:

A. Mass as quantity of material.

B. Relation of volume to size.

C. Density as a characteristic property.

D. Proportionality to mass.

E. Inverse proportionality to volume.

F. Computation of density.

2. To develop an instrument for evaluating student understanding of density.
Although one-on-one interviews are preferred for most accurate probe of student understanding, time constraints often render them impractical. Treagust found that diagnostic tests, specifically including multiple-choice tests, could be useful in evaluating student misconceptions in science. ${ }^{5}$ A preliminary test was developed from the results of a survey of the literature and from one-on-one interviews with seven high school students. Face validity was established by three university faculty and six precollege science teachers.

The instrument includes 11 multiplechoice questions. ${ }^{6}$ Each of the elements of density is tested with one or two questions. One item from the Third International Mathematics and Science Study (TIMSS) is included to allow assessment of student facility with proportional reasoning. ${ }^{7}$ This item and one testing the computation of density require numerical answers. Three items (see Figure 1) are adapted from questions posed by Kanim in the context of electric charge density. ${ }^{8}$

\section{Data Collection and Analysis}

Data were collected early in the 20002001 school year. Results were analyzed for measures of central tendency and distribution of responses for the entire test, for each question, and for each of the six elements of density. Written explanations for two questions were analyzed in detail.

\section{Sample Population}

Rather than follow a single population over an extended period, this cross-age study documents student understanding of density across three educational levels at a single moment in time. The total sample 
A straight, uniform board is cut into three differently sized pieces such that the sum of the lengths of the two shorter pieces is equal to the length of the longest piece. Each piece has identical width and thickness.

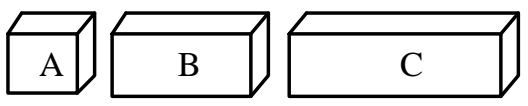

1. Which piece has the greatest volume?

2. Which piece has the greatest density?

3. Which piece has the greatest mass?
A. Piece A
C. Piece C
B. Piece B
D. They are all the same.

Figure 1: Items 1-3 of the instrument.

population of 787 students includes 328 middle school students (MS), 271 high school students (HS) and 188 college students (CS), drawn from five institutions in Southern California, a region that is ethnically and economically diverse.

Each level included two sets of students. Set MS1 includes sixth and eighth graders, while MS2 consists of eighth graders. HS1 is composed of students in a coordinated science course. HS2 consists of students enrolled in Advanced Placement or honors biology courses. CS1 consists of students in an introductory survey of physics for nonscience majors. CS2 includes students in six sections of an upper division biology course for preservice elementary teachers, and was the only set in which students were taught by different instructors.

Density was explicitly taught in only two of the courses. MS1 had completed a one-week lesson on density approximately two weeks prior to the test. CS1, taught by one of the study authors, had completed approximately two hours of instruction on density and buoyancy. HS1 were in the second year of a two-year coordinated Earth, physical and life science program that includes instruction on density in the first year. Some CS2 students had recently completed an upper division physical science course that covers density.

\section{RESULTS}

Table I presents a summary of the responses of 787 students to the survey instrument. The average number correct is for responses to the ten questions on density. The percentage of correct responses on the TIMSS proportional reasoning question is shown for comparison. (Group MS2 took a version of the instrument in which the TIMSS question was omitted.) Also shown is the fraction of students answering at least 8 of the density-related questions correctly.

Not surprisingly, the more advanced students tended to score higher. However, differences were less than expected, and overall success rates are low. Even the students in CS2 who had taken an upper division physical science course answered an average of only 5.7 questions correctly ( $N$ $=22$ ); the average for the remaining 126 students in this group was 5.3 correct.

In addition to the overall success rate, we examined response patterns for items testing each of the elements of density. In the discussion below, percentages refer to results from all student sets combined.

The two questions relating to mass as quantity of material elicited very different rates of success. In question 3 (see Figure 1 ), the object with the largest mass also has the largest volume. The other question involves the more difficult concept of conservation of mass in the context of a sealed balloon whose volume decreases with a temperature change. The former question was answered correctly by $71 \%$ of the students as compared to only $36 \%$ for the balloon question. The balloon question had a strong distracter, with $36 \%$ responding that the mass would decrease.

Two questions addressing the relation of volume to size were answered correctly by $75 \%$ and $52 \%$ of the students. Whereas question 1 compares three objects of different sizes, the other question addresses the volume of a balloon as it changes size. Although these rates are relatively high, only 39\% answered both items correctly. About $20 \%$ of the students answered that the 


\begin{tabular}{|c|c|c|c|}
\hline Population & Mean (St. Dev.) & \% correct, TIMSS item & \% with 8+ correct \\
\hline $\begin{array}{c}\text { MS 1 (N = 155) } \\
\text { Grades 6 \& 8 }\end{array}$ & $3.26(1.58)$ & $50 \%$ & $\sim 1 \%$ \\
\hline $\begin{array}{c}\text { MS 2 }(N=173) \\
\text { Grade 8 }\end{array}$ & $3.98(1.56)$ & n/a & $2 \%$ \\
\hline $\begin{array}{c}\text { HS 1 }(N=153) \\
\text { Grade 9/10 }\end{array}$ & $4.57(1.79)$ & $66 \%$ & $6 \%$ \\
\hline $\begin{array}{c}\text { HS 2 }(N=118) \\
\text { AP / Honors Bio }\end{array}$ & $6.18(2.06)$ & $79 \%$ & $23 \%$ \\
\hline $\begin{array}{c}\text { CS 1 }(N=40) \\
\text { Gen. Ed. Physics }\end{array}$ & $7.48(1.66)$ & $68 \%$ & $52 \%$ \\
\hline $\begin{array}{c}\text { CS 2 }(N=148) \\
\text { Bio for Teachers }\end{array}$ & $5.34(1.98)$ & $69 \%$ & $14 \%$ \\
\hline
\end{tabular}

Table I: Student responses to the density instrument for several populations.

volume of the balloon was the same after the change in size. ${ }^{9}$

Two items address the concept of density as a characteristic property. Each was answered correctly by about $30 \%$ of the students. In question 2, 31\% of the students answered that the density of the largest piece would be the greatest, and $25 \%$ answered that the smallest piece would have the greatest density. In the other item, which asks students to compare the density of a small diamond chip to that of the whole diamond, 36\% answered that the density of the chip would be smaller.

The item addressing the variation of density with mass was answered correctly by $60 \%$ of the students. In contrast, the two items addressing the inverse variation of density with volume had success rates around $30 \%$. In both problems, many students chose answers consistent with the idea that the object's density is related to its size. For example, $32 \%$ of the students answered that a balloon's density decreased as it underwent thermal contraction. In addition, 32\% answered that the balloon's density would remain the same. Written explanations given in support of this answer suggested that the density of air is fixed:

The amount of air doesn't matter, all air will have the same density.

\section{CONCLUSIONS}

The overall correct response rate of $47 \%$ suggests that many of the students in this study had not developed a complete understanding of density. Even among the relatively sophisticated populations in the college and high school honors courses, the overall success rate was low and very few of the students answered 8 or more questions correctly. Particularly noteworthy is the low performance of the preservice teachers, despite the completion in some cases of an upper division course in physical science.

Many student responses show a consistent pattern of associating each of the quantities mass, volume, and density with size. These responses can be interpreted as illustrating an inability to distinguish between these related concepts. An alternative interpretation is based on the work of Stavy and Tirosh, who assert that many students answer questions in mathematics by appealing to intuitive rules:

...the rule 'More A - More B' is... the common core to many reported apparent misconceptions. ${ }^{10}$

All of the qualitative questions on this survey can be answered using this rule, which would lead to some correct and some incorrect answers. For example, in questions $1-3$, the most common responses 
were those stating that the largest piece of wood has the largest volume, density and mass, respectively. Application of the intuitive rule yields a correct response in two of the three questions but fails in the third. This is characteristic of intuitive rules: they make sense and they work in a large variety of situations. Use of the intuitive rule could also account for the low success rate on questions testing the concept of inverse variation of density with volume.

Approximately $66 \%$ of the students in this study answered the TIMSS question correctly, as compared to $71 \%$ of the students among the international student sample in the TIMSS study. Although these numbers are not directly comparable, because all the TIMSS students were in their final year of secondary education, they do suggest that the students in this study are not unusually deficient in their ability to apply proportional reasoning skills.

Our results also support the widespread finding that the ability to solve a quantitative problem does not necessarily imply a conceptual understanding. Performance on the quantitative density question was often unrelated to performance on the remainder of the survey. During the development of the survey instrument, a high school physical science class used a prototype as a pre- and post-instruction assessment. In this class, there was a small increase in student performance, but the entire change was due to an increase in the number of students answering the quantitative question correctly. The total number of correct answers for the qualitative density questions was exactly the same before and after extensive lab-based instruction on density.

This preliminary study has several limitations. Since the subjects are taken from different populations, a longitudinal analysis of student understanding is not possible. The components of density are investigated with only one or two questions each, and a multiple-choice instrument may limit responses. Any differences between sets may be the result of group selection or teacher effect, as in the case of results from CS 1 and CS 2.
Additional work to validate the instrument is planned. However, the results certainly provoke important questions. For example, do science standards introduce density at an appropriate point in the curriculum? By contributing to systematic assessment of student understanding, we expect the instrument to serve as a tool for researchers and practitioners.

\section{ACKNOWLEDGMENTS}

The authors thank Dr. Galen Carlson, who provided essential feedback, and all who reviewed and tested the instrument.

\footnotetext{
${ }^{1}$ National Research Council, National Science Education Standards, Washington, DC, National Academy Press (1996).

2 See, for example, E. Gennaro, “Assessing Junior High Students' Understanding of Density and Solubility," School Sci. and Math, 81 (1981), D.L. Shepherd and J.W. Renner, "Student Understandings and Misunderstandings of States of Matter and Density Changes," School Sci. and Math., 63, 415-522 (1982), J. W. McKinnon, "Earth Science, Density, and the College Freshman,” J. Geol. Educ., 19, 218-220 (1971), M. G. Hewson and P. W. Hewson, "Effect of instruction using students' prior knowledge and conceptual change strategies on science learning,” J. Res. in Sci. Teaching, 20(8), 731-743 (1983).

${ }^{3}$ S. Kanim, Ph.D. dissertation, University of Washington, 1999 (unpublished), M. Loverude, Ph.D. dissertation, University of Washington, 1999 (unpublished).

${ }^{4}$ B. Inhelder and J. Piaget, The Growth of Logical Thinking from Childhood to Adolescence, Basic Books (1958), p. 36.

${ }^{5}$ D. F. Treagust, "Development and use of diagnostic tests to evaluate students' misconceptions in science,” Int. J. Sci. Ed., 10, 159-169 (1988).

${ }^{6}$ The instrument can be found at http://chaos.fullerton.edu/ mloverud ${ }^{7}$ TIMSS Released Item Set, Final Year of Secondary School, MSL Item D7.

${ }^{8}$ See ref. 3.

${ }^{9}$ See also C. Kautz, Ph.D. dissertation, University of Washington, 1999 (unpublished).

${ }^{10}$ R. Stavy and D. Tirosh, How Students (Mis)Understand Science and Mathematics: Intuitive Rules, Albany, New York: Teachers College Press, (2000), p. 2.
} 